\title{
PAPER
}

\section{Development of a software tool for eliminating nonlinear distortion}

\author{
Jun Hamada, Yoshinobu Kajikawa* and Yasuo Nomura \\ Department of Electoronics, Faculty of Engineering, Kansai University, \\ 3-3-35, Yamate-cho, Suita, 564-8680 Japan
}

(Received 15 October 2002, Accepted for publication 4 March 2003 )

\begin{abstract}
In this paper, we present the effectiveness of a software tool for eliminating nonlinear distortions of loudspeaker systems through some experiments. The nonlinear distortions affect the sound quality of loudspeaker systems. We have presented some identification methods of loudspeaker systems and some compensation methods of nonlinear distortions. However, the software tool for compensating the distortions has not been realized yet. We therefore develop the software tool. Experimental results show that the 2nd- and 3nd-order nonlinear distortions of a loudspeaker system can be reduced in the range of $10[\mathrm{~dB}]$ to $20[\mathrm{~dB}]$ by the developed tool when noise level is below $70[\mathrm{dBA}]$.
\end{abstract}

Keywords: Loudspeaker, Nonlinear distortions, Volterra filter, Eliminating distortions

PACS number: 43.58.Ry, 43.60.-c [DOI: 10.1250/ast.24.186]

\section{INTRODUCTION}

The fundamental principle of loudspeaker systems has never been changed since the invention. The loudspeaker systems have so complex structure to transform electric signal into mechanical vibration and to radiate acoustic wave so that the loudspeaker system produces linear and nonlinear distortions. These distortions consequently deteriorate the sound quality. Recently, small-sized loudspeaker systems for desktop have come into wide use as personal computers (PC) advance. Since these size gets smaller than general speaker systems inevitably, the level of nonlinear distortions get bigger generally. Furthermore, it has been reported that the nonlinear distortions have influence for auditory sense in high-sampling audio [1,2]. Hence, the importance of eliminating the nonlinear distortion has been increasing in recent years.

Identifying the linear and nonlinear elements of loudspeaker systems and designing a nonlinear inverse system are essential to eliminate these distortions. We have demonstrated the effectiveness of an elimination method using Volterra filters [3]. In this method, the Volterra kernels of a target loudspeaker system are identified by adaptive Volterra filters [4] and the nonlinear distortion is eliminated by a nonlinear inverse system using the identified Volterra kernels [5]. By the way, since the tendency listening to music with PC has been increasing, user's demands of eliminating the nonlinear distortion with PC have been increasing. However, a software tool for eliminating the nonlinear distortion has not been realized yet. We have therefore developed the software tool, which can identify loudspeaker systems, design the corresponding nonlinear inverse system, and compensate the nonlinear distortions. Experimental results demonstrate the effectiveness of the software tool.

\section{ELIMINATION METHODS OF NONLINEAR DISTORTION USING VOLTERRA FILTER}

In this chapter, we explain how to eliminate the nonlinear distortion of a loudspeaker system using Volterra filters.

\subsection{Discrete Volterra Series}

Nonlinear systems such as loudspeaker systems can be modeled by using the Volterra series expansion. Since this paper treats the 3rd-order element and assumes that the Volterra kernels have a finite memory length $N$, the inputoutput relation of the systems is represented by

*e-mail: kaji@joho.densi.kansai-u.ac.jp 


$$
\begin{aligned}
y(n)= & \sum_{k_{1}=0}^{N-1} h_{1}\left(k_{1}\right) x\left(n-k_{1}\right) \\
& +\sum_{k_{1}=0}^{N-1} \sum_{k_{2}=0}^{N-1} h_{2}\left(k_{1}, k_{2}\right) x\left(n-k_{1}\right) x\left(n-k_{2}\right) \\
& +\sum_{k_{1}=0}^{N-1} \sum_{k_{2}=0}^{N-1} \sum_{k_{3}=0}^{N-1} h_{3}\left(k_{1}, k_{2}, k_{3}\right) x\left(n-k_{1}\right) x\left(n-k_{2}\right) x\left(n-k_{3}\right)
\end{aligned}
$$

where $x(n)$ and $y(n)$ are the discrete input and output signals, respectively; $h_{1}\left(k_{1}\right), h_{2}\left(k_{1}, k_{2}\right)$, and $h_{3}\left(k_{1}, k_{2}, k_{3}\right)$ are the 1st-, 2nd-, and 3rd-order discrete Volterra kernels, respectively. The Volterra kernels satisfy the symmetry property; therefore, these kernels are invariant regard less of the order of its terms, without loss of generality. For example, the 3rd-order Volterra kernel has the property as

$$
\begin{aligned}
& h_{3}\left(k_{1}, k_{2}, k_{3}\right)=h_{3}\left(k_{1}, k_{3}, k_{2}\right)=h_{3}\left(k_{2}, k_{1}, k_{3}\right) \\
& \quad=h_{3}\left(k_{2}, k_{3}, k_{1}\right)=h_{3}\left(k_{3}, k_{1}, k_{2}\right)=h_{3}\left(k_{3}, k_{2}, k_{1}\right)
\end{aligned}
$$

\subsection{Discrete Fourier Transform of Volterra Series}

Discrete Fourier transform (DFT) of Eq. (1) is given by

$$
\begin{aligned}
Y(m)= & H_{1}(m) X(m) \\
& +A_{1}\left[H_{2}\left(m_{1}, m_{2}\right) X\left(m_{1}\right) X\left(m_{2}\right)\right] \\
& +A_{2}\left[H_{3}\left(m_{1}, m_{2}, m_{3}\right) X\left(m_{1}\right) X\left(m_{2}\right) X\left(m_{3}\right)\right]
\end{aligned}
$$

where $X(m)$ and $Y(m)$ are the $N$ point DFTs of $x(n)$ and $y(n)$, respectively; $H_{1}(m), H_{2}\left(m_{1}, m_{2}\right)$, and $H_{3}\left(m_{1}, m_{2}, m_{3}\right)$, which are called the 1st-, 2nd-, and 3rd-order Volterra Frequency Responses (VFR), are those of $h_{1}\left(k_{1}\right), h_{2}\left(k_{1}, k_{2}\right)$, and $h_{3}\left(k_{1}, k_{2}, k_{3}\right)$, respectively. Furthermore, the VFR also satisfy the symmetry property due to the symmetry of the Volterra kernels. For example, the 3rd-order VFR has the property as

$$
\begin{aligned}
& H_{3}\left(m_{1}, m_{2}, m_{3}\right)=H_{3}\left(m_{1}, m_{3}, m_{2}\right)=H_{3}\left(m_{2}, m_{1}, m_{3}\right) \\
& \quad=H_{3}\left(m_{2}, m_{3}, m_{1}\right)=H_{3}\left(m_{3}, m_{1}, m_{2}\right)=H_{3}\left(m_{3}, m_{2}, m_{1}\right)
\end{aligned}
$$

Moreover, the VFR satisfies the conjugate symmetry property. For example, the 3rd-order VFR has the property as

$$
H_{3}\left(m_{1}, m_{2}, m_{3}\right)=H_{3}{ }^{*}\left(N-m_{1}, N-m_{2}, N-m_{3}\right)
$$

$A_{1}$ and $A_{2}$ are called the reduction operators. The reduction operator has the role of mapping a function with multidimensional dependent variables to a function with onedimensional variables [6]. $A_{1}$, which maps a function with two-dimensional dependent variables to that with onedimensional variables, is represented as

$$
\begin{aligned}
Y(m) & =A_{1}\left[Y_{2}^{\prime}\left(m_{1}, m_{2}\right)\right] \\
& =\frac{1}{N} \sum_{m_{1}+m_{2}=m \text { or } m+N} Y_{2}^{\prime}\left(m_{1}, m_{2}\right)
\end{aligned}
$$

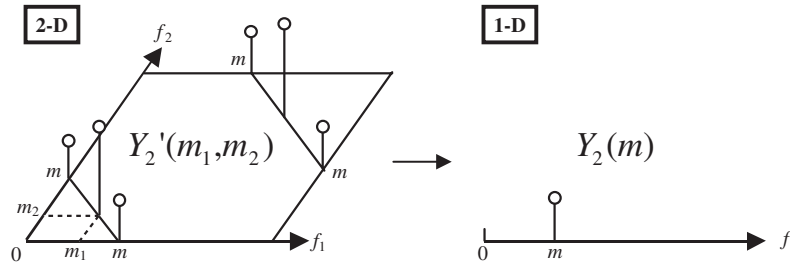

Fig. 1 Reduction operator.

where $Y(m)$ and $Y_{2}^{\prime}\left(m_{1}, m_{2}\right)$ are the function with one- and two-dimensional dependent variables, respectively. Figure 1 shows the reduction operator. In Eq. (6), the spectra $Y_{2}{ }^{\prime}\left(m_{1}, m_{2}\right)$ at the frequencies satisfying $m_{1}+m_{2}=m$ or $m+N$ are summed, so that the spectrum $Y(m)$ at the frequency $m$ can be obtained. Similarly, $A_{2}$, which maps a function with three-dimensional dependent variables to that with one-dimensional variables, is represented as

$$
\begin{aligned}
Y(m) & =A_{2}\left[Y_{3}^{\prime}\left(m_{1}, m_{2}, m_{3}\right)\right] \\
& =\frac{1}{N^{2}} \sum_{m_{1}+m_{2}+m_{3}=m \text { or } m+N \text { or } m+2 N} Y_{3}{ }^{\prime}\left(m_{1}, m_{2}, m_{3}\right)
\end{aligned}
$$

where $Y_{3}^{\prime}\left(m_{1}, m_{2}, m_{3}\right)$ are the function with three-dimensional dependent variables. In Eq. (7), the spectra $Y_{3}^{\prime}\left(m_{1}, m_{2}, m_{3}\right)$ at the frequencies satisfying $m_{1}+m_{2}+$ $m_{3}=m$ or $m+N$ or $m+2 N$ are summed, so that the spectrum $Y(m)$ at the frequency $m$ can be obtained.

\subsection{Identification of Loudspeaker System Using Frequency Response Method [7]}

To obtain the 3rd-order VFR, three sinusoidal waves, whose frequencies are $m_{1}, m_{2}$, and $m_{3}\left(m_{1}<m_{2}<m_{3}\right)$, are input into a nonlinear system. Then, the VFR is determined by substituting spectra of input signal $X\left(m_{1}\right), X\left(m_{2}\right)$ and $X\left(m_{3}\right)$ and output signal for

$$
H_{3}\left(m_{1}, m_{2}, m_{3}\right)=\frac{Y\left(m_{1}+m_{2}+m_{3}\right)}{X\left(m_{1}\right) X\left(m_{2}\right) X\left(m_{3}\right)} \frac{N^{2}}{a}
$$

where the coefficient $a$ is the number of the symmetry of the VFR. Moreover, the 3rd-order VFR is determined by repeating this procedure at various frequencies. Since sinusoidal waves are input, this method has much superior identification accuracy. 


\subsection{Nonlinear Inverse System}

Figure 2 shows the structure of a nonlinear inverse system to eliminate the 2nd- and 3rd-order distortions. In

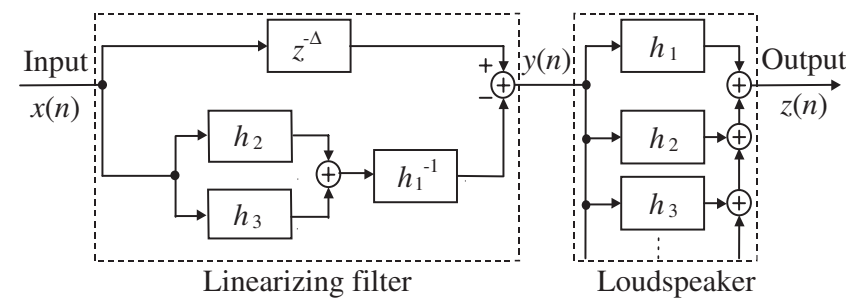

$h_{1}: 1^{\text {st }}$-order Volterra kernel $h_{1}^{-1}: 1^{\text {st }}$-order inverse filter $h_{2}: 2^{\text {nd }}$-order Volterra kernel $z^{-\Delta}:$ Delay

$h_{3}: 3^{\text {rd }}$-order Volterra kernel

Fig. 2 The structure of a nonlinear inverse system.
Fig. 2, the linear inverse filter $h_{1}^{-1}$ satisfies $h_{1} \cdot h_{1}^{-1}=z^{-\Delta}$. To design the nonlinear inverse system, the linear inverse filter $h_{1}^{-1}$ and the 2nd- and 3rd-order Volterra kernels $h_{2}, h_{3}$ are needed. Furthermore, those accuracies affect the performance of the nonlinear inverse system.

\section{SOFTWARE TOOL FOR ELIMINATING NONLINEAR DISTORTION}

In this chapter, we explain how to use the developed software tool. We purpose eliminating the distortions of small-sized loudspeaker system for PC by software. The main menu is shown in Fig. 3(a). Functions of the software tool are as follows;

- Identification of loudspeaker system

- Design of linear inverse filter

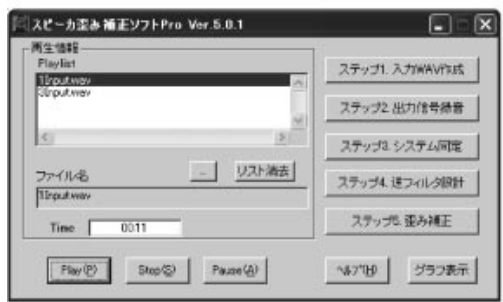

(a) Main menu.

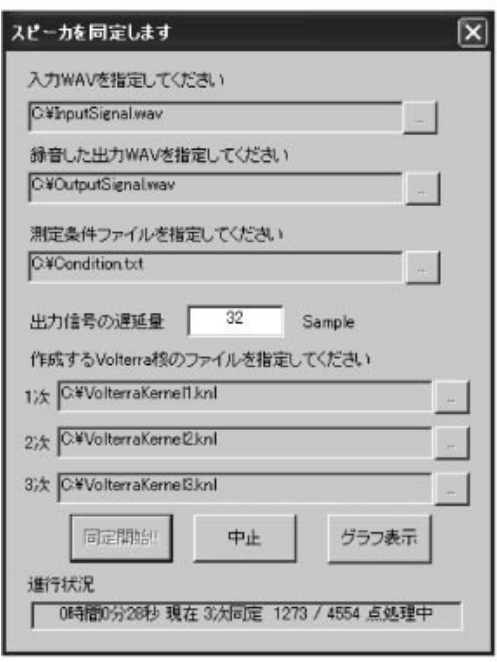

(d) Identification.

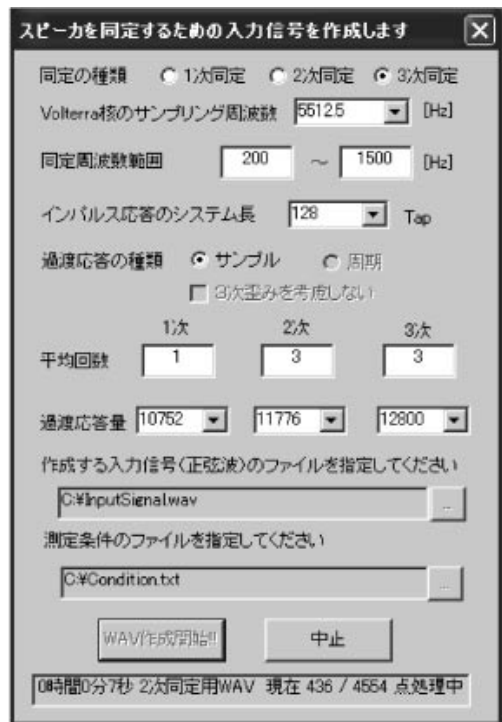

(b) Making input signal.

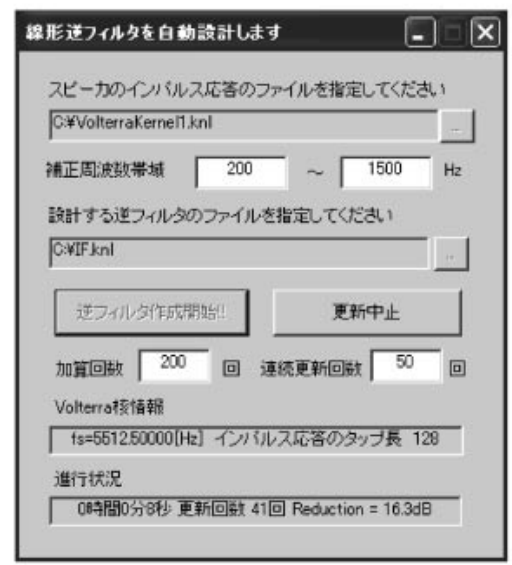

(e) Making linear inverse filter.

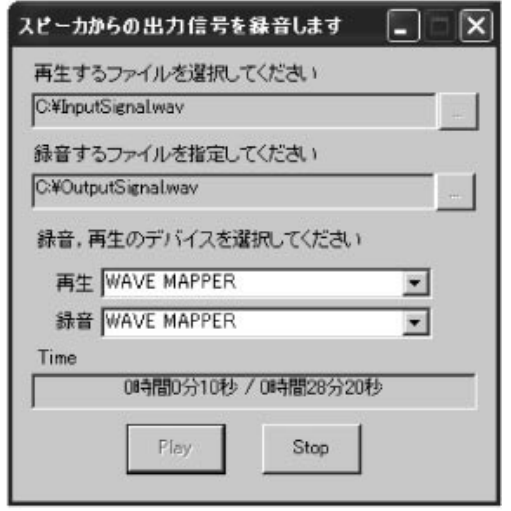

(c) Recording.

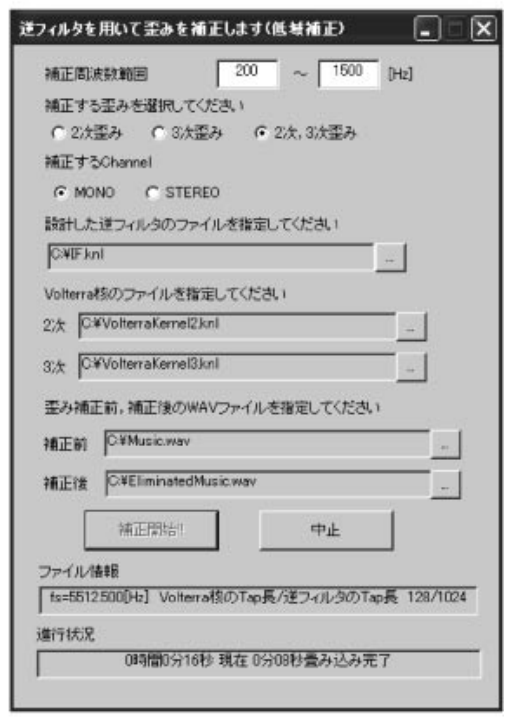

(f) Elimination.

Fig. 3 Functions of the tool. 
- Eliminating distortions

- Playback WAV files, and so on

\subsection{Making Input Signal (Fig. 3(b))}

We can make an input signal for the identification as a WAV file. We need to set up the order of Volterra kernels, frequency range, sampling frequency, tap length, the number of average, and so on. Furthermore, the record conditions are made as text file. Moreover, the sampling frequency can be flexibly set in a frequency range lower than $44,100[\mathrm{~Hz}]$ and the maximum frequency to be able to eliminate the nonlinear distortion becomes consequently below half of the sampling frequency.

\subsection{Recording Output Signal (Fig. 3(c))}

We can set up playback and record devices freely and record the output signal of a loudspeaker system.

\subsection{Identification of Volterra Kernels (Fig. 3(d))}

By setting up the WAV files of the input signal made in Sect. 3.1 and the output signal recorded in Sect. 3.2, we can obtain the Volterra kernels.

\subsection{Design of Linear Inverse Filter (Fig. 3(e))}

We can design the linear inverse filter by using the 1storder Volterra kernel in Sect. 3.3. By using FrequencyDomain Summational Normalized Block LMS (FDSNBLMS) algorithm [8], only the filter coefficients within a target frequency range is updated. Futhermore, the stepsize parameter is set up automatically and the design of the linear inverse filter is finished when the reduction, which is defined as Eq. (9), becomes maximum.

Reduction $=10 \log _{10}\left[\sum_{m=m_{1}}^{m_{2}} D(m)^{2} / \sum_{m=m_{1}}^{m_{2}}\{D(m)-Y(m)\}^{2}\right]$

$D(m)$ : Spectrum of desired signal.

$Y(m)$ : Spectrum of signal thorough linear inverse filter.

Eliminating frequency range: $m_{1}-m_{2}[\mathrm{~Hz}]$

\subsection{Eliminating Distortions (Fig. 3(f))}

By setting up a playback WAV file, the linear inverse filter, and the Volterra kernels, we can make the corresponding WAV file to make the 2nd- and 3rd-order distortions eliminate.

\section{EXPERIMENTS OF ELIMINATING NONLINEAR DISTORTIONS}

Next, we experiment with eliminating the 2nd- and 3rdorder nonlinear distortions by the developed software. Tables 1 and 2 shows the measurement conditions and the identification conditions. First, we measure the Volterra
Table 1 Measurement conditions.

\begin{tabular}{lr} 
CPU & Pentium IV 2.0AGHz \\
Main memory & DDR-SDRAM 1GB \\
OS & Windows XP \\
Sound card & On board \\
Microphone & MM-MC5 (SANWA SUPPLY) \\
Loudspeaker & MM-SP80SV (SANWA SUPPLY) \\
\hline
\end{tabular}

Table 2 Identification conditions.
Sampling frequency

Frequency range

Tap length of 1st-order kernel

Tap length of 2nd-order kernel

Tap length of 3rd-order kernel
$5,512.5[\mathrm{~Hz}]$

$200-1,500[\mathrm{~Hz}]$

$$
256
$$

128

128 kernels of a loudspeaker system and design the linear inverse filter. Next, we play back sinusoidal waves for a test and the corresponding output waves for the nonlinear inverse system, then record the output signals of the loudspeaker system. Finally, we calculate the frequency responses of harmonic and intermodulation elements by using Eqs. (10)-(14).

$$
\begin{aligned}
H_{2 m}(m) & =\frac{Y(2 m)}{X(m)} \\
H_{m_{2} \pm m_{1}}(m) & =\frac{Y\left(m_{2} \pm m_{1}\right)}{X\left(m_{2}\right)} \\
H_{3 m}(m) & =\frac{Y(3 m)}{X(m)} \\
H_{m_{2} \pm 2 m_{1}}(m) & =\frac{Y\left(m_{2} \pm 2 m_{1}\right)}{X\left(m_{2}\right)} \\
H_{m_{3} \pm\left(m_{1}+m_{2}\right)}(m) & =\frac{Y\left(m_{3} \pm\left(m_{1}+m_{2}\right)\right)}{X\left(m_{3}\right)}
\end{aligned}
$$

In Eqs. (11) and (13), $m_{1}$ is kept at $215[\mathrm{~Hz}]$ and $m_{2}$ is swept in the frequency range of $258[\mathrm{~Hz}]$ to $1,464[\mathrm{~Hz}]$. Similarly, $m_{1}$ and $m_{2}$ are kept at $215[\mathrm{~Hz}]$ and $258[\mathrm{~Hz}]$ respectively, and $m_{3}$ is swept in the frequency range of $301[\mathrm{~Hz}]$ to $1,464[\mathrm{~Hz}]$ in Eq. (14). Here, the noise level is $50[\mathrm{dBA}]$ in both the identification and elimination and the playback sound pressure level is $92[\mathrm{~dB}]$. Noise level is defined as follows.

$$
\text { Noise level }[\mathrm{dBA}]=10 \log _{10} \frac{P_{A}^{2}}{P_{0}^{2}}
$$

$P_{A}^{2}$ : A-weighted sound pressure level.

$P_{0}^{2}$ : Standard sound pressure level $(20[\mu \mathrm{Pa}])$.

Figure 4(a)-(h) shows that the frequency responses of a loudspeaker system and the 2nd- and 3rd-order nonlinear distortions before and after elimination. We also calculate the frequency response of the 4th-order harmonic distortion as shown in Fig. 4(i) because the nonlinear inverse system 


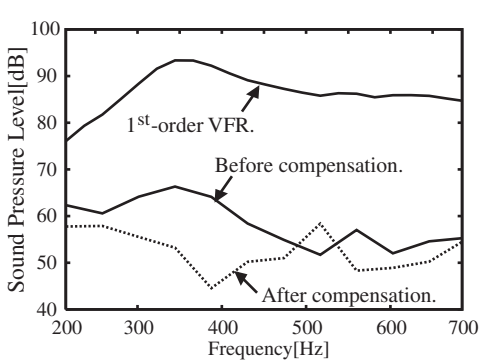

(a) $2 m$ element

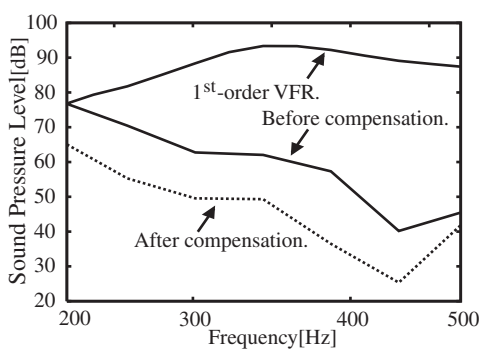

(d) $3 m$ element

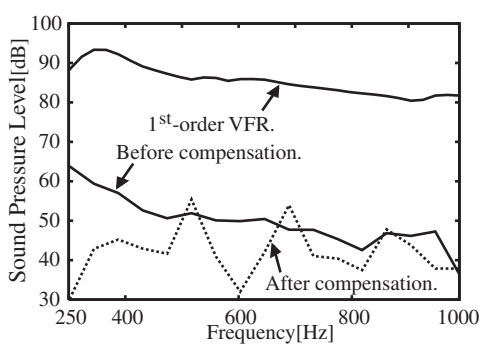

(g) $m_{3}+m_{1}+m_{2}$ element

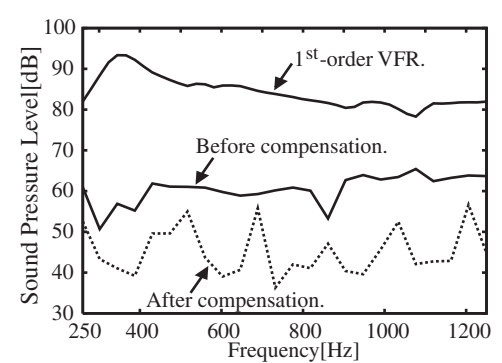

(b) $m_{2}+m_{1}$ element

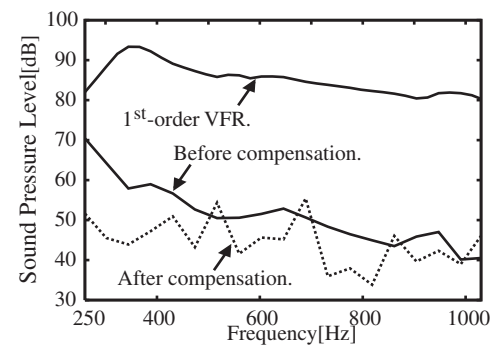

(e) $m_{2}+2 m_{1}$ element

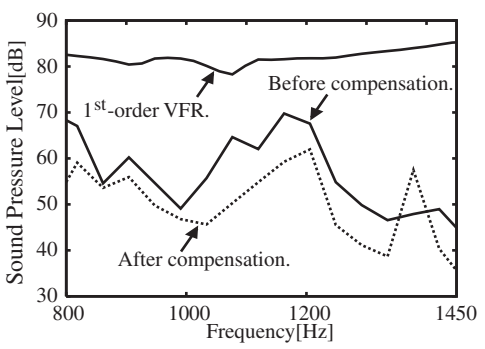

(h) $m_{3}-\left(m_{1}+m_{2}\right)$ element

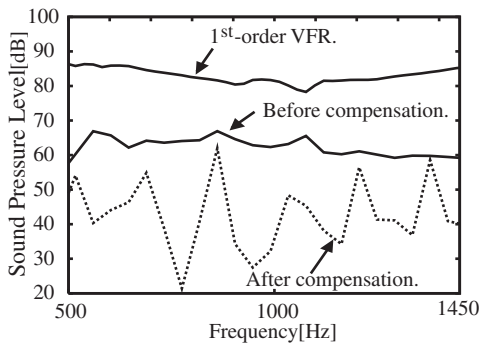

(c) $m_{2}-m_{1}$ element

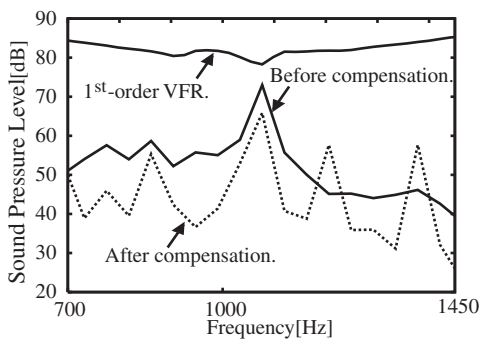

(f) $m_{2}-2 m_{1}$ element

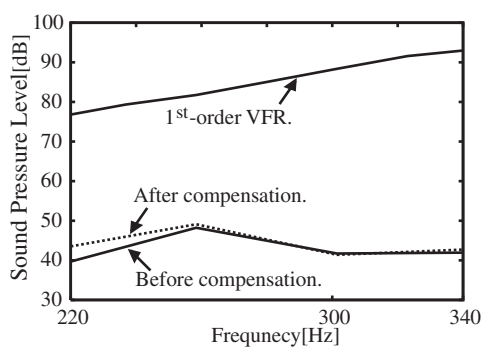

(i) $4 m$ element

Fig. 4 Frequency responses of a loudspeaker system and the nonlinear distortions before and after elimination.

in Fig. 2 may make the higher-order distortions increase. In Fig. 4, $x$-axis and $y$-axis express the swept frequency and the sound pressure level respectively. It can be seen from Fig. 4 that the levels of the 2nd- and 3rd-order nonlinear distortions are reduced in the range of $10[\mathrm{~dB}]$ to $20[\mathrm{~dB}]$ compared with before elimination over the whole frequency band. Moreover, all nonlinear distortion levels after compensation are lower than the background noise level.
The increase of the 4th-order harmonic distortion is also a little. These results demonstrate the effectiveness of the developed software tool.

Next, we experiment with eliminating the 2nd- and 3rdorder nonlinear distortions using the Volterra kernels identified under some noisy environments. Here, we use white Gaussian noise to make noisy environments. Table 3 shows the effects of eliminating nonlinear distortions. In

Table 3 Effectiveness of eliminating nonlinear distortions.

\begin{tabular}{|c|c|c|c|c|c|c|c|c|}
\hline \multirow{2}{*}{$\begin{array}{c}\text { Noise level } \\
\text { Elements }\end{array}$} & \multicolumn{2}{|c|}{$50[\mathrm{dBA}]$} & \multicolumn{2}{|c|}{$60[\mathrm{dBA}]$} & \multicolumn{2}{|c|}{$70[\mathrm{dBA}]$} & \multicolumn{2}{|c|}{$80[\mathrm{dBA}]$} \\
\hline & Points & Effect [dB] & Points & Effect [dB] & Points & Effect [dB] & Points & Effect [dB] \\
\hline $2 m_{1}$ & $1 / 12$ & 5.9 & $3 / 12$ & 5.3 & $2 / 12$ & 7.4 & $3 / 12$ & 2.6 \\
\hline$m_{2}+m_{1}$ & $0 / 24$ & 15.4 & $0 / 24$ & 13.8 & $0 / 24$ & 10.4 & $2 / 24$ & 7.0 \\
\hline$m_{2}-m_{1}$ & $0 / 24$ & 19.6 & $0 / 24$ & 17.2 & $1 / 24$ & 12.3 & $6 / 24$ & 9.0 \\
\hline $3 m_{1}$ & $0 / 7$ & 13.0 & $1 / 7$ & 11.8 & $0 / 7$ & 9.7 & $2 / 7$ & 7.0 \\
\hline$m_{2}+2 m_{1}$ & $4 / 19$ & 6.7 & $3 / 19$ & 5.9 & $7 / 19$ & 1.4 & $13 / 19$ & -2.1 \\
\hline$m_{2}-2 m_{1}$ & $2 / 19$ & 8.2 & $5 / 19$ & 5.3 & $8 / 19$ & 0.7 & $14 / 19$ & -5.8 \\
\hline$m_{3}+m_{1}+m_{2}$ & $4 / 18$ & 7.8 & $4 / 18$ & 5.1 & $9 / 18$ & 0.9 & $13 / 18$ & -4.4 \\
\hline$m_{3}-\left(m_{1}+m_{2}\right)$ & $1 / 18$ & 6.9 & $1 / 18$ & 6.7 & $8 / 18$ & 4.1 & $9 / 18$ & -2.0 \\
\hline Average effect & \multicolumn{2}{|c|}{$10.4[\mathrm{~dB}]$} & \multicolumn{2}{|c|}{$8.9[\mathrm{~dB}]$} & \multicolumn{2}{|c|}{$5.9[\mathrm{~dB}]$} & \multicolumn{2}{|c|}{$0.3[\mathrm{~dB}]$} \\
\hline
\end{tabular}


Table 3, 'Points' is the number of points increasing in the distortion per the number of evaluating points; 'Effect' is an average eliminated quantity to all evaluating points per element and 'Average effect' is an average eliminated quantity to all elements per 'Noise level'. The negative value for 'Effect' means the increase of the distortion. It can be seen from Table 3 that the effect is smaller as the increase of noise level. These results demonstrate nonlinear distortions of a loudspeaker system can be reduced fully when the noise level is below $70[\mathrm{dBA}]$. Therefore, this software is available for general environment, such as home use of personal computers with general devices, because $70[\mathrm{dBA}]$ is the same as the sound pressure level of the telephone ring.

In this experiment, it took about 40 minutes to identify a loudspeaker system, 3 minutes to design the linear inverse filter, and about 5 minutes to compensate a 4minutes WAV file, respectivelly.

\section{CONCLUSIONS}

In this paper, we have developed a software tool for eliminating nonlinear distortions. The experimental results have demonstrated the effecitiveness of the software tool. Effectiveness of eliminating nonlinear distortions of loudspeaker system in high-sampling audio are a subject to study in the future.

\section{ACKNOWLEDGEMENT}

This work was supported in part by Grants-in-Aid for Scientific Research No. 14750320 from the Ministry of Education, Culture, Sports, Science and Technology.

\section{REFERENCES}

[1] K. Ashihara and S. Kiryu, "A measurement of non-linearity distortions of loudspeaker by using sweeping band-elimination filter and average response method," J. Acoust. Soc. Jpn. (J), 56, 69-77 (2000).

[2] K. Ashihara and S. Kiryu, "Influence of expanded frequency band of signals on non-linear characteristics of loudspeakers," $J$. Acoust. Soc. Jpn. (J), 56, 549-555 (2000).

[3] M. Schetzen, Volterra and Wiener Theories of Nonlinear Systems (Krieger, Florida, 1989).

[4] V. J. Mathews, "Adaptive polynomial filters," IEEE Signal Process. Mag., 8, 10-26 (1991).

[5] Y. Kajikawa and Y. Nomura, "An online design method of nonlinear inverse system by adaptive Volterra filter," IEICE Trans., J82-A, 1-10 (1999).

[6] S. Ichikawa, S. Tokoro, S. Kawasaki, S. Hagihara and T.
Hagiwara, "Calculating method of reduction of high-order Volterra functional," IEICE Trans., J65-A, 77-84 (1982).

[7] M. Tsujikawa, Y. Kajikawa and Y. Nomura, "Identification of third-order Volterra kernels by frequency response method and its application to loudspeaker systems," IEICE Trans., J84-A, 1333-1345 (2001).

[8] M. Tsujikawa, Y. Kajikawa and Y. Nomura, "Frequency domain adaptive Volterra filters using summational complex NLMS algorithm," Tech. Rep. IEICE, DSP2000-124, pp. 51-58 (2000).

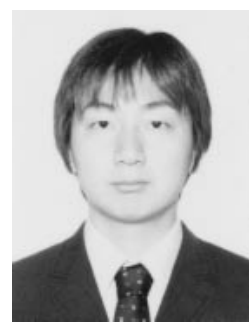

Jun Hamada was born in Osaka, Japan, on Jun. 29, 1978. He received the B.E. and M.E. degrees in electronic engineering from Kansai University, Osaka, Japan in 2001 and 2003, respectively. He conducted research on nonlinear signal processing for his master thesis. He currently joins Matsushita AVC Multimedia Software Co. Ltd.

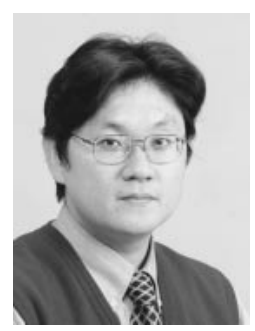

Yoshinobu Kajikawa was born in Hyogo, Japan, on Jan. 11, 1969. He received the B.E. and M.E. degrees in electronic engineering from Kansai University, Osaka, Japan, and the D.E. degree in communication engineering, graduate school of engineering from Osaka University in 1991, 1993, and 1997, respectively. He joined Fujitsu Ltd. in 1993. In 1994, he joined Kansai University, where he is now an Associate Professor of Electronic Engineering. His research interests are in digital signal processing, nonlinear signal processing, adaptive filtering, audio and communication systems, and intelligent robot. Dr. Kajikawa is a Member of the Institute of Electronics, Information and Communication Engineers (IEICE), the Institute of Electrical and Electronics Engineers, Inc. (IEEE), the European Association for Signal Processing, and the Information Processing Society of Japan. He received the Young Engineer Award in 1997 from IEICE of Japan and the Awaya Prize in 2001 from ASJ.

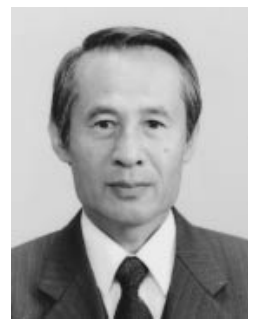

Yasuo Nomura received the B.E. and M.E. degrees in Communication Engineering from Osaka University, Osaka, Japan. He joined Matsushita Electric Industrial Ltd. He also has the D.E. degree. In 1970, he became a research student at Osaka University, where in 1975 he became a lecturer at Kansai University, where he was promoted to an associate professor and a professor of Electronic Engineering. His research interests are in CAD of electro-acoustic transducers and artificial intelligence. Dr. Nomura is a Member of the Institute of Electronics, Information and Communication Engineers (IEICE), the Institute of Electrical and Electronics Engineers, Inc. (IEEE), and the Information Processing Society of Japan. 